East African Medical Journal Vol. 77 No. 5 May 2000

SARCOMAS OF THE HEAD AND NECK AT KENYATTA NATIONAL HOSPITAL

M. L. Chindia, BDS, MSc, FFDRCSI, Lecturer, Oral and Maxillofacial Surgery, Faculty of Dental Sciences, University of Nairobi;

S.M. Swaleh, BDS, Dental Officer and P.M. Godiah, BDS, Dental Officer, Dental Unit, Kenyatta National Hospital., P.O. Box 20723, Nairobi, Kenya.

Request for reprints to: Dr. M. L. Chindia, Faculty of Dental Sciences, P.O. Box 19676, Nairobi, Kenya.

\title{
SARCOMAS OF THE HEAD AND NECK AT KENYATTA NATIONAL HOSPITAL
}

\section{L. CHINDIA, S.M. SWALEH and P.M. GODIAH}

\begin{abstract}
Objective: To determine the pattern of occurrence of sarcomas afflicting the neck and craniofacial region.

Design: A retrospective study (1982-1991).

Setting: Cancer Registry, Kenyatta National Hospital, Nairobi, Kenya.

Method: Examination was performed of the cancer records in the registry over the period 1982 to 1991 inclusive. Histopathological types of the sarcomas were then analysed to indicate the numbers that occurred per year; and also the pattern of occurrence according to age groups, gender and site.

Results: Of the 10,897 whole body neoplasms, 985 were sarcomas. Among these sarcomas, $160(16 \%)$ were located in the neck and craniofacial region. The histopathological types of the neoplasms included Kaposi's sarcoma (39\%), osteosarcoma (23\%), rhabdomyosarcoma (21 \%), fibrosarcoma (13\%), chondrosarcoma (two per cent), malignant fibrous histiocytoma (one per cent) and dermatofibrosarcoma protuberans (one per cent).

Conclusion: The results of this investigation, in concordance with those of other series worldwide, indicate that the various sarcomas afflicting the neck and craniofacial area are generally rare.
\end{abstract}

\section{INTRODUCTION}

On a worldwide basis, reports on the patterns of occurrence of sarcomas of the neck and craniofacial region are limited to the presentations of specific case studies and reviews. Yet these neoplasms comprise those malignancies that often cause not only the most devastating morbidity but also very rapid mortality among afflicted patients. Sarcomas refer to a wide variety of rare malignant tumours that arise from the primitive mesoderm including connective tissue, endothelium, mesothelium and nerve cell components. The poor prognosis of most patients with a sarcoma of the head and neck region is due to its aggressive behaviour, and difficulty in obtaining local control(1).

So far, limited epidemiological studies have shown little information causally linking environmental factors to the occurrence of sarcomas in man. Furthermore, no firm advances have been made towards the effective treatment of nearly all these neoplasms for long-term survival. The emergence of the human immunodeficiency virus (HIV) infection has precipitated an increased occurrence of Kaposi's sarcoma of the neck and craniofacial region. It is therefore important that baseline information regarding the pattern of occurrence of these neoplasms is reported frequently from all regions of the world. This article presents the occurrence of various sarcomas of the neck and craniofacial region over a ten-year period as recorded at the Cancer Registry of the Kenyatta National Hospital in Kenya.

\section{MATERIALS AND METHODS}

The Kenyatta National Hospital (KNH) in Nairobi, is Kenya's main medical teaching centre and referral hospital serving a population of approximately 26 million. In 1968, a cancer registry was established at the institution by the World Health Organisation through the International Agency for Research on Cancer(2).

The pathology records in the Cancer Registry at this centre were examined for all cases of whole body malignant neoplasms, whole body sarcomas and specifically neck and craniofacial sarcomas occurring over the period 1982 to 1991 (10 years). The neck and craniofacial sarcomas were then analysed to indicate numbers occurring per year; and also according to age, sex and site of occurrence.

\section{RESULTS}

Out of 10,897 whole body neoplasms, 985 were sarcomas. Among the sarcomas afflicting the whole body 160 neck and craniofacial neoplasms were identified. 
Table 1

Yearly profile of whole body neoplasms and sarcomas

\begin{tabular}{lccc}
\hline Year & $\begin{array}{l}\text { No. of whole body } \\
\text { neoplasms }\end{array}$ & $\begin{array}{l}\text { No. of whole body } \\
\text { sarcomas }\end{array}$ & $\begin{array}{l}\text { No. of neck and } \\
\text { craniofacial sarcomas }\end{array}$ \\
\hline 1982 & 1786 & 125 & 23 \\
1983 & 1715 & 124 & 16 \\
1984 & 1750 & 135 & 29 \\
1985 & 1244 & 111 & 15 \\
1986 & 939 & 77 & 13 \\
1987 & 311 & 82 & 14 \\
1988 & 733 & 69 & 9 \\
1989 & 656 & 70 & 10 \\
1990 & 856 & 90 & 160 \\
\hline Total & 907 & 102 & 10 \\
\hline
\end{tabular}

\section{Table 2}

Yearly profile of types of neck and craniofacial sarcomas

\begin{tabular}{|c|c|c|c|c|c|c|c|c|}
\hline Year & OS & RMS & FBS & CHS & MFH & DFSP & $\mathrm{KS}$ & Yearly total \\
\hline 1982 & 7 & 1 & 2 & 0 & 0 & 1 & 12 & 23 \\
\hline 1983 & 6 & 5 & 3 & 0 & 0 & 0 & 2 & 16 \\
\hline 1984 & 8 & 7 & 7 & 2 & 0 & 0 & 5 & 29 \\
\hline 1985 & 4 & 3 & 6 & 0 & 0 & 0 & 6 & 19 \\
\hline 1986 & 3 & 5 & 2 & 0 & 0 & 0 & 5 & 15 \\
\hline 1987 & 2 & 3 & 1 & 0 & 0 & 1 & 6 & 13 \\
\hline 1988 & 5 & 3 & 0 & 0 & 0 & 0 & 6 & 14 \\
\hline 1989 & 0 & 3 & 0 & 0 & 1 & 0 & 8 & 12 \\
\hline 1990 & 1 & 1 & 0 & 1 & 0 & 0 & 6 & 9 \\
\hline 1991 & 1 & 3 & 0 & 0 & 0 & 0 & 6 & 10 \\
\hline Total & 37 & 34 & 21 & 3 & 1 & 2 & 62 & 160 \\
\hline
\end{tabular}

Key: OS=Osteorsarcoma; $\mathrm{CHS}=$ Chondrosarcoma; DFSP=Dermatofibrosarcoma protuberance; $\mathrm{RMS}=\mathrm{Rhaddomyosarcoma}$ MFH=Malignant fibrous histiocytoma; FBS=Fibrosarcoma; KS=Kaposi's sarcoma

Table 3

Distribution of sarcoma types according to the age range and sex

\begin{tabular}{|c|c|c|c|c|c|c|c|}
\hline \multirow[t]{2}{*}{ Tumour type } & \multicolumn{5}{|c|}{ Age range (yrs) } & \multicolumn{2}{|c|}{ Sex distribution } \\
\hline & $0-10$ & $11-20$ & $21-30$ & $31-40$ & $41+$ & M & $\mathrm{F}$ \\
\hline $\mathrm{KS}$ & 16 & 5 & 16 & 4 & 21 & 45 & 17 \\
\hline OS & 4 & 12 & 9 & 3 & 9 & 21 & 16 \\
\hline RMS & 22 & 9 & - & 1 & 2 & 18 & 16 \\
\hline FBS & 5 & 3 & 1 & 2 & 10 & 16 & 5 \\
\hline CHS & - & 1 & - & 1 & 1 & 2 & 1 \\
\hline $\mathrm{MFH}$ & - & 1 & - & - & - & 1 & - \\
\hline DFSP & - & 1 & - & - & 1 & 2 & - \\
\hline Total & 47 & 32 & 26 & 11 & 44 & 105 & 55 \\
\hline
\end{tabular}


Table 4

Distribution of sarcomas of the neck and craniofacial region according to site

\begin{tabular}{lrr}
\hline Site & No. of sarcomas & $\%$ \\
\hline Neck region & 33 & 20.63 \\
Floor of mouth & 7 & 4.38 \\
Mandibular & 24 & 15.00 \\
Maxillary & 29 & 18.13 \\
Nasoethmoidal & 13 & 8.13 \\
Zygormatic complex & 1 & 0.63 \\
Parotid & 12 & 7.50 \\
Mastoid & 5 & 3.13 \\
Ocular & 18 & 11.25 \\
Auricular & 4 & 2.50 \\
Temporal area & 1 & 0.63 \\
Scalp and calvarium & 13 & 8.13 \\
\hline Total & 160 & 100 \\
\hline
\end{tabular}

Histopathologically, Kaposi's sarcoma was the commonest subtype $(39 \%)$ followed by osteosarcoma (23\%), rhabdomyosarcoma (21\%), fibrosarcoma (13\%), chondrosarcoma (two per cent), malignant fibrous histiocytoma (one per cent) and dermatofibrosarcoma protuberans (one per cent). Characteristics of the various sarcoma types are presented in Tables 1-4.

\section{DISCUSSION}

Generally, neck and craniofacial mesenchymal malignant neoplasms are rare. In the present study, these neoplasms comprise only about $15 \%$ of all the sarcomas afflicting the whole body. Remarkably, lesions occurring in the jaws and floor of the mouth constituted more than $40 \%$ of all the sarcomas afflicting the neck and craniofacial region. It is also worthy of note in this series that most sarcomas occurred in the youngest age group in the first decade of life and the oldest age group above 40 years. In addition, it is evident that males are afflicted more commonly than females with an overall ratio of approximately $2: 1$.

As shown in Table 1, the yearly pattern of occurrence of sarcomas in the neck and craniofacial region was somewhat variable with a range of nine to 29 lesions. The decreasing trend of the numbers of sarcomas diagnosed after the mid 1980s may be attributed to the fact that other centres than KNH within Nairobi and regionally had started active participation in histopathological examination and reporting. However, no specific reason was apparent as to why the number of cases analysed in 1987 was exceptionally low.

Notably, the diagnosis of Kaposi's sarcoma (KS) occurring in the neck and craniofacial region generally showed a consistent trend over the study period. This neoplasm has been described as being one of the commonest malignant tumours in Africa, accounting for about nine per cent of all neoplasms in the indigenous population(3). The neoplasm is the commonest cancer associated with the HIV infection, occurring in up to one in four AIDS patients with a male to female ratio of 20:1(4). Indeed, KS is now epidemic in countries where HIV is most prevalent(5). The present findings indicate a contrasting male to female ratio of nearly $3: 1$ which may be a reflection of a regional variation.

Among the soft tissue sarcomas, apart from KS, rhabdomyosarcoma (RMS) was diagnosed most frequently and our findings revealed a particularly high occurrence of this neoplasm in the age group 0 to 10 years thereby corroborating other published series. These neoplasms have a relative predilection of the head and neck region(8). RMS may be defined as a malignant tumour of the rhabdomyoblasts and it is estimated to comprise $12 \%$ to $56 \%$ of all solid malignant tumours in the paediatric age group (9).

Other malignant tumours of soft tissue origin included the fibrosarcoma (FBS), malignant fibrous histocytoma (MFH) and dermatofibrosarcoma protuberans (DFSP). FBS is a malignant tumour whose occurrence in the head and neck is rare, accounting for less than five per cent of fibrosarcomas of all sites(10). The intraoral or paraoral FBS may arise in any location, but is most commonly associated with the cheek, maxiliary sinus, pharynx, palate, lip and periosteum of the maxilla or mandible(7). If clinical data for children and young adults are combined, FBS involving the head and neck make up $18 \%$ to $20 \%$ of the cases(11). Among the paediatric cases, the histopathology varies somewhat from that occurring in adults and has a markedly different clinical behaviour(12). The most striking finding in the present study was the male to female ratio of about 3 to 1 of FBS occurring in the neck and craniofacial region. Remarkably, five of the 21 cases were children aged up to 10 years.

Over the ten-year period, only two cases of DFSP and one of MFH had been diagnosed. DFSP is a rare mesenchymal tumour of the skin whose incidence is low: $0.06 \%$ of all malignant neoplasms and $1.17 \%$ of all soft tissue sarcomas(13). This neoplasm is said to be an intermediate grade sarcoma that arises in the dermis of the trunk, proximal extremities and less commonly in the head and neck area of adults(14).

MFH is believed to be a rare tumour of the head and neck(15). About three per cent of reported cases of MFH have arisen in the head and neck(16). Although classically defined as a soft tissue sarcoma, MFH can also originate in bone(17). The most common and difficult problem in the differential diagnosis is the distinction of MFH from other malignant neoplasms showing a comparable degree of cellular pleomorphism(18). The origin of this tumour is controversial as it was initially proposed that the tissue histiocyte was acting as a facultative fibroblast and that this explained the apparent bimodal cell population(19). Indeed, Sohail et al(20) have observed that the diagnosis of MFH may only be mandatory where immunohistochemical or ultrastructural studies have excluded other tumours (such as spindle cell squamous carcinoma, malignant melanoma) that can appear identical on conventional microscopic examination. 
Of the neoplasms primarily arising from bone tissue, osteosarcoma (OS) and chondrosarcoma (CHS) were identified. In this study, most of the osteosarcomas occurred in the 10 to 30 years age group (Table 3). This age group is generally lower than has been reported in other studies(21). The average age of onset of the tumours of the jaws and craniofacial bones is 10 to 20 years later than for skeletal lesions; and the histopathological variables are more favourable, distant metastases occur less frequently and survival rates are higher(21-23). Between five and $10 \%$ of all osteosarcomas are found in the bones of the skull, with the majority found in the jaw bones(22). Our results show that the number of lesions located in the maxilla and mandible (33\%) is consistent with other reports. Notably, there was a slight preponderance of OS among males over females in the ratio of 1.3:1.

As would be expected, based on evidence elsewhere(6), chondrosarcomas were found to have been particularly rare in this series. Only three lesions had been diagnosed over the ten-year period. In general, CHS occurs more often in males, in a ratio of about $2: 1$; and there are no pathognomonic signs or symptoms presented(7). In view of the small number of lesions identified in the current study, no specific observations could be made regarding this neoplasm.

It is evident from this investigation that over $70 \%$ of the sarcomas afflicting the neck and craniofacial region occur earlier than the fourth decade of life. The public needs with respect to cancer prevention, detection and treatment should therefore, be of profound interest among health providers and adminstrators. While prevention closely relates to the avoidance of known aetiological factors, early detection of neoplastic change requires not only an efficient clinical and laboratory medical care system but also one that is easily accessible and affordable by the public. Unfortunately, these are tenets that remain grossly inadequate in many economically underdeveloped societies.

Undoubtedly, current innovations in cancer research in general are oriented towards the establishment of the regional pattern of distribution of various neoplastic lesions. In this way it may be possible to, more precisely, evaluate the environmental factors that could be associated with the occurrence of any specific neoplastic lesion. Furthermore, the ongoing explosive laboratory and clinical investigations including the biological behaviour patterns of neoplasms at the molecular and cytogenetic levels may hold key answers regarding oncogenesis in general. Certainly, the feasibility of studies such as these may only be accomplished in prospective protocols where patients are comprehensively evaluated as they present. However, the retrospective analysis of existing archival material remains important since this exercise indeed offers the baseline information upon which specific prospective analytical protocols may arise.

Notwithstanding the comparatively small numbers of sarcomas vis-a-vis other malignancies afflicting the neck and craniofacial area, their often grave prognosis has to be of tremendous concern at all times. For the effective treatment, good histologic differentiation of the primary tumour is of particular importance. Therefore, the comprehensive documentation of the numbers of sarcoma entities that occur globally and the monitoring of their biological behaviour patterns at presentation and during treatment needs to be encouraged if future advances in the effective control of these often lethal neoplasms is to be realised sooner than later.

\section{ACKNOWLEDGEMENTS}

We are most grateful to the Ethics and Research Committee of the Kenyatta National Hospital for authorising the execution of this study; and to Professor A. Nyong'o for his permission enabling us to access the cancer registry records. We are also most thankful to Miss Carolyne M. Imbayi for preparing this manuscript and to Dr. K.A. Wakoli and Dr. F.L. Ndungu for their support and encouragement.

\section{REFERENCES}

1. Sanroman, J.F., Alonso Del Hoyo Jr, Diaz F.J., Fil-Die J.L., Monje F., Naval L., Alamillos F.J., Dean A. and Rubio P. Sarcomas of the head and neck. Brit. J. Oral Maxillofac. 1992; 30:115-118.

2. Cook P. Meeting on sources of cancer statistics. 4th-8th November 1968. World Health Organization International Agency for Research on Cancer: EP1/S. StaV8.

3. Woolf, N. Disorders Related to the Immune System. In: Cell Tissue and Disease. The Basis of Pathology. 2nd ed. Bailliene Tindall. London. Pg. 146-148, 1986.

4. Soames, J.V. and Southam, J.C. Infections of the Oral Mucosa. In: Oral Pathology. 2nd ed. Oxford University Press. Oxford. Pg.196, 1993.

5. Ziegler, J.L. and Katongole-Mbidde, E. Kaposi's Sarcoma in childhood: An analysis of 100 cases from Uganda and Relationship to HIV Infection. Int. J. Cancer. 1996; 65: 200-203.

6. Hackney, F.L., Aragon, S.B., Aufdemorte, T.B., Holt, G.R. and Van Sickels, J.E.: Chondrosarcoma of the jaws: Clinical findings, histopathology and treatment. Oral Surg. Oral Med. Oral Path. 1991; 71: 139-143.

7. Shafer, W.G., Hine, M.K. and Levy, B.M. Benign and Malignant tumours of the oral cavity. In: A Textbook of Oral Pathology. 4th ed. W.B. Saunders Company. Philadelphia. Pg. 86-229, 1983.

8. Doval, D.C., Kannan, V., Acharya, R.S. Mukherjee, G., Shenoy, A.M. and Bapsy, P.P. Rhabdomyosarcoma of the tongue. Brit. J. Oral. Maxillofac. Surg. 1994; 32:183-6

9. Brookes, C.N. and Van Velzen, C. Rhabdomyosarcoma, Presenting as a facial swelling in a child. A case report and review of the literature. Brit. J Oral Maxillofac. Surg. 1990; 28: 73-79.-

10. Scott, S.M., Reiman, H.M., Prichard, D.J. and Ilstrup, D.M. Soft tissue fibrosarcomas: A clinicopathologic study of 132 cases. Cancer 1989; 64: $925-933$.

11. Dehner, L.P. Tumours in the Mandible and Maxilla in Children.Cancer. 1973; 31: 112-120.

12. Bang, G, Baardisen, R. and Gilhuus-Moe, O. Infantile Fibrosarcoma in the Mandible: Case Report. J. Oral Path. Med. 1989; 18:339-343.

13. Smola,M.G., Sover, H.P. and Scharnagl. Surgical Treatment of Dermatofibrosarcoma Protuberans. A retrospective study of 20 cases with review of literature. Eur J. Surg. Oncol. 1991; 17: 447-453.

14. Laskin W.B. Dermatofibrosarcoma Protuberans. CA Cancer J. 1992; 42: 116-125.

15. Blitzer, A., Lawson, W., Zak, K.G., Biller, H.F. and Som, M.L. Clinicalpathological determinants in prognosis of fibrous histiocytomas of head and neck. The laryngoscope 1981: 2053-2067.

16. Ireland, A.J., Eveson, J.W. and Leopard, P.J. Malignant Fibrous Histiocytoma: A report of two cases arising in sites of previous irradiation. Brit. J. Oral Maxillafac. Surg. 1988; 26: 221-227.

17. Bras, J. Malignant fibrous histiocytomas of the oral soft tissues. Oral Surg. Oral Med. Oral Path. 1987; 64:57-67.

18. Weis, S.W. and Enzinger, F.M. Malignant Fibrous histiocytoma: An analysis of 200 cases. Cancer 1978; 41:2250-2266.

19. Besly, W., Wiesenfeld, D., Kleid, S. Allan, P. and Poker, I. Malignant fibrous histiocytoma of the maxilla - A report of two cases. Brit. J. Oral Maxillofac. Surg. 1993; 31: 45-48.

20. Sohail, D., Derr, R., Simpson, R.H.W. and Babajews, A.V. Malignant fibrous histiocytoma of the mandible: The importance of accurate histopathological diagnosis. Brit. J. Oral. Maxillofac. 1995, 33:166-168.

21. Regezi, J.A., McClatchey, K.D. and Crissman, J.D. Osteosarcoma and Chondrosarcoma of the jaws: Immunohistochewichcorrelation. Oral Surg. 1987; 4: 302-307.

22. Batsakis, J.G. Osteogenic and chondrogenic sarcomas of the jaws. Ann. Oto. Rhinol. Laryngol. 1987; 9: 474-475.

23. Russ J.E. and Jesse, R.H. Management of osteosarcoma of the maxilla and mandible. Amer. J Surg. 1980; 140: 572-57 . 